\title{
Fear of Missing Out Scale - A Nationwide Representative CAWI Survey in Poland
}

\author{
Anna Jupowicz-Ginalska \\ (iD) ORCID: 0000-0002-7016-0427 \\ UNIVERSITY OF WARSAW, POLAND \\ Małgorzata Kisilowska \\ D ORCID: 0000-0001-5733-5424 \\ UNIVERSITY OF WARSAW, POLAND
}

\author{
Tomasz Baran \\ ORCID 0000-0003-2922-7551 \\ UNIVERSITY OF WARSAW, POLAND \& ARIADNA \\ RESEARCH PANEL \\ Aleksander Wysocki
ARIADNA RESEARCH PANEL \\ Justyna Jasiewicz \\ (D) ORCID 0000-0002-8869-0792 \\ UNIVERSITY OF WARSAW, POLAND
}

\begin{abstract}
Fear Of Missing Out (FOMO) is a growing problem that arouses interest from scientists, media and recipients. Therefore, finding a universal way to calculate the scale of the phenomenon can not only become a common tool for estimating the extent of FOMO occurrence in any country, but also can be considered for managing this phenomenon. The article presents the results of measuring the FOMO scale in Poland in 2018. The project applied a Computer Assisted Web Interview survey of the representative sample $(\mathrm{N}=1060)$ of Poland's internet users aged 15 years plus. Three groups of 'FOMO level' characteristics were determined (age was the most influential variable). The original method of recalculation of the FOMO scale results from figures to percentage data was developed in order to present the FOMO scale index. The FOMO items showed good internal consistency and reliability Cronbach's $\alpha=.89$, thus complementing previous international studies.
\end{abstract}

KEYWORDS: fear of missing out, FOMO scale, representative survey, social media studies, social media users.

\section{INTRODUCTION}

Fear of Missing Out (FOMO) increasingly raises interest among researchers, society and media. The academic literature is dominated by analyses combining this phenomenon with the technological context, the use of social media or the so-called problematic internet use (PIU). However, so far only a few projects have determined its scale within a given population - be it a nation, the internet 
or social media users. We carried out the FOMO research on a representative sample in order to determine the scale of this phenomenon in Poland. This, in the context of research literature in the Web of Science's index, was one of the first representative surveys about FOMO.

The study has the following goals: 1) the determination of the range of FOMO occurrence among the Poland's internet users aged 15 years and over; 2) the presentation of basic demographic and behavioural characteristics of people experiencing low, medium and high FOMO, and 3) the demographics and behavioural characteristics of people experiencing high FOMO.

We decided not to make any hypothesis because our study is of an explorative nature. In the following parts of the text, we present and discuss the findings of our research on the representative sample of users of Poland's internet (social media in particular) who are aged 15 years or more. We take into consideration not only the methodology and results, but also the process of converting the FOMO scale results into percentage data, and the development of the FOMO index. We finish with the conclusions and further research needs.

Selected information and data were presented in a special report, addressed to the public, the media and NGOs (Jupowicz-Ginalska et al., 2018). This report had educational and promotion functions, and therefore only limited data were offered (basic methodological and quantitative information). This article discusses the methodology of the study, with particular attention put on the process of recalculating the figures into percentage results of the FOMO scale for the sample, as well as the results themselves, comparing the latter to other FOMO projects (see also literature review below).

We repeated the research on FOMO in Poland in 2019, using the same methodology (it has proved to have good internal consistency and reliability of Cronbach's $\alpha$, which means that our method is a stable tool that allows researchers to estimate FOMO levels). The results of this research were presented in the second report (Jupowicz-Ginalska et al., 2019). However, its data do not appear in this article and we inform about them for the sake of reliability in presenting the effects of our work.

\section{THEORETOCAL BACKGROUND}

Analysis of FOMO-related publications revealed that Przybylski et al. (2013) composed the most cited definition of FOMO, which states that FOMO is 'a pervasive apprehension that others might be having rewarding experiences from which one is absent (...) the desire to stay continually connected with what others are doing'. Although the definition does not relate FOMO to new technologies, the permanence of the connection is realised in the 2010s through the 
internet and social media in particular (Auverset et al. 2016; Chotpitayasunondh \& Douandglas, 2016; Elhai et al., 2016; Hetzet al. 2015), as a risk or negative consequence of social media activities. Our project uses the FOMO definition by Przybylski et al. (2013).

The FOMO phenomenon has been explored mostly with quantitative methodology, with a few studies based on qualitative or mixed methods. Among 62 articles for the years 2013-2018 indexed in the Web of Science, there are only 4 projects based on a representative sample. The founding study of Przybylski et al. (2013) was realised partially ( 1 of 3 stages of the project) on a representative sample ( $\mathrm{N}=2079)$ of working age adults (aged 22-65 years) in Great Britain. FOMO proved to be the factor explaining relations between need satisfaction, well-being and social media use by the respondents. This stage of the study was conducted as an online survey with the FOMO scale developed during stage 1, and aimed to find the demographic, motivational and well-being factors related to FOMO. Overall, FOMO was negatively correlated with age, and partially correlated with gender (young men were more endangered by FOMO). The overall study focused more on a psychological aspect of FOMO, i.e. the motivational, emotional and behavioural correlations. The study did not present an in-depth analysis of demographic characteristics of FOMO in the sample population.

In next two studies by chronology, both involving representative samples FOMO was not the main subject of analysis, but a factor contributing to digital stress. The first was the 2013 American study of 1801 adult (aged 18 years and over) Americans, which involved both internet and non-internet users (Hampton et al. 2016). The study focused on the relation between digital media usage and stress, and FOMO was referred to only as one of the characteristics of social media users. The second used a survey of 1557 German internet users (aged 14-85 years) and focused on psychological health effects and motivational origins of digital stress (Reinecke et al., 2017). Only three items of the questionnaire measured FOMO. The phenomenon was found to be positively related to communication load and internet multitasking.

The fourth and the last project was an American online survey on a representative sample of 518 social media users (aged 18-65), which recognised FOMO as the factor most influencing social media fatigue.

As the FOMO phenomenon has, so far, been analysed mostly as a factor of digital stress or problematic internet behaviour, the present study is to be the first (dated on 2018) which has FOMO as the key research topic, defines FOMO levels for the population, and provides detailed demographic characteristics of 'high-FOMO' people.

In the context research the most commonly used FOMO research tool is the 10-statement set FOMO scale, that Przybylski et al. (2013) developed. 
Among 62 Web of Science indexed articles, 2013-2018, 30 quantitative surveys (and articles) used the questionnaire in its original version. However, there are also studies with the FOMO scale extended to 18 questions (Alt, 2015; Alt, 2017) or modified in other ways (adapted, shortened, etc.) - in 7 projects (Yin et al., 2015; Aarestad \& Eide, 2017; James et al., 2017; Salim et al., 2017; Bailey et al., 2018; Dhir et al., 2018; Franchina et al., 2018; Scalzo \& Martinez, 2018). Foreign language versions also exist: Spanish (Fuster et al., 2017), Italian (Casale et al., 2018) and Chinese (Xie et al., 2018).

\section{METHODS}

The diagnostic survey method and the Computer Assisted Web Interview (CAWI) technique were applied in the study.

\section{RESEARCH INSTRUMENT}

Taking into account its popularity and reliability verified in many studies (as mentioned above), we decided to implement Przybylski's at al. (2013) FOMO scale in its original version (see Table 2). We translated it into Polish and then included a more extensive questionnaire, the aim of which was to analyse the specificity of FOMO in Poland in a multithreaded manner. This means that FOMO scale was the most important part of this extended questionnaire, which also included the following thematic sections: social media usage; reasons for using social media; positive and negative effects of using social media; building personal image by using social media; vulnerability to marketing activities in social media; psychological and physical status of the respondents.

The extended questionnaire consisted of 499 questions, 475 of which were arranged in the form of a Likert scale (three questions used a bipolar ordinal scale, the remainder used a five-point scale). Demographic characteristics (gender, age, place of residence) and social attitudes were also included.

The extended questionnaire was developed in January 2018. Journalism students of the University of Warsaw participated in a pilot test in February 2018, followed which minor corrections were applied. The final CAWI survey was conducted in March 18-28, 2018, on a nationwide probability random-quota sample of the internet users aged $15+(\mathrm{N}=1060)$ selected from Poland's nationwide Ariadna research panel. The quotas on sex, age, and the size of the place of residence were based on how the population of Poland is represented among the nation's internet users. Participants completed a secure online survey based on software optimized for use on desktop computers, tablets, and mobile devices. In this text, we focus only on the implementation of the FOMO scale in Poland. 


\section{RESEARCH SAMPLE CHARACTERISTICS - DEMOGRAPHIC VARIABLES}

The final sample of respondents consisted of the internet users $\mathrm{N}=1060$, age ranging from 15 to 83 years, $\mathrm{MEAN}=37$ years, $\mathrm{SD}=14$ years, gender ratio of of $50.3 \%$ females and $49.7 \%$ males. The response rate was $65 \%$. The specific demographics of the sample are presented in Table 1.

Table 1. Research sample characteristics - demographic variables

\begin{tabular}{|c|c|c|c|}
\hline Sample characteristics & Total $\mathrm{N}=1060$ & $\begin{array}{c}\text { Male } 50 \%, \\
\mathbf{n}=527\end{array}$ & $\begin{array}{c}\text { Female } 50 \% \text {, } \\
n=533\end{array}$ \\
\hline \multicolumn{4}{|c|}{ Age years (frequencies and numbers) } \\
\hline mean_SD & $37 \%(14.0)$ & $38.4 \%(14.3)$ & $35.4 \%(13.4)$ \\
\hline $15-24$ & $24 \%(252)$ & $21 \%(110)$ & $27 \%(143)$ \\
\hline $25-34$ & $28 \%(294)$ & $27 \%(143)$ & $28 \%(151)$ \\
\hline $35-44$ & $21 \%(221)$ & $22 \%(114)$ & $20 \%(107)$ \\
\hline $45-54$ & $13 \%(141)$ & $14 \%(75)$ & $13 \%(66)$ \\
\hline $55+$ & $14 \%(151)$ & $16 \%(85)$ & $12 \%(66)$ \\
\hline \multicolumn{4}{|c|}{ Place of residence (frequencies and numbers) } \\
\hline village & $35 \%(373)$ & $35 \%(186)$ & $35 \%(187)$ \\
\hline small / middle size town & $36 \%(382)$ & $35 \%(186)$ & $37 \%(196)$ \\
\hline city / metropolis & $29 \%(305)$ & $30 \%(155)$ & $28 \%(150)$ \\
\hline \multicolumn{4}{|c|}{ Education level (frequencies and numbers) } \\
\hline primary & $14 \%(146)$ & $15 \%(80)$ & $12 \%(65)$ \\
\hline secondary & $46 \%(482)$ & $46 \%(241)$ & $45 \%(241)$ \\
\hline high & $40 \%(432)$ & $39 \%(205)$ & $43 \%(227)$ \\
\hline
\end{tabular}

Source: Authors

\section{METHODOLOGY OF DATA ANALYSIS}

We developed the FOMO scale index aggregating all the 10 components used in the measure as a set, to precisely determine FOMO intensity among the Poland's internet users, and to present FOMO in figures. We asked the respondents to indicate their level of agreement on a 5-point Likert scale, where 0 value represented 'not at all true about me' to 4 - 'extremely true about me'. Descriptive statistics of the FOMO 10 -item battery set for the internet population's basic demographic variables are presented in Table 2, and the analysis of the statistical significance of the FOMO 10-item battery set for the internet population's basic demographic variables is presented in Table $3(\mathrm{p}<0.05)$. Please note that light grey marking means the subgroup indicated has a significantly higher response value (dark grey marking means that the subgroup in question has a significantly lower response value). 
Table 2. Descriptive statistics of FOMO 10-item battery - demographic variables

\begin{tabular}{|c|c|c|c|c|c|c|c|c|c|c|c|}
\hline $\bar{\Xi}$ & high & 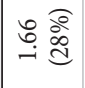 & 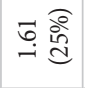 & 악 & 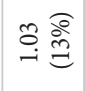 & $\begin{array}{l}\infty \\
\infty \\
\rightarrow \infty\end{array}$ & $\stackrel{\infty}{\stackrel{\infty}{\stackrel{2}{d}}}$ & 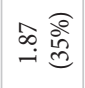 & 守 产 & 苗 & 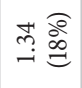 \\
\hline 当 & secondary & g. & 苍 & 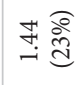 & oิ & 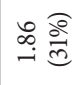 & in & के & 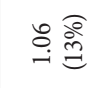 & 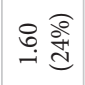 & 운 \\
\hline & primary & 范 & ت্ & 萧 & $\stackrel{\infty}{=} \underset{\stackrel{9}{g}}{\stackrel{d}{=}}$ & 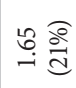 & $\stackrel{3}{-\stackrel{9}{g}}$ & 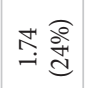 & 곯 & $\stackrel{8}{\stackrel{i}{\stackrel{d}{d}}}$ & ผึ \\
\hline 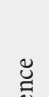 & $\begin{array}{l}\text { large town } \\
\text { metropolis }\end{array}$ & 늘 & $\stackrel{\substack{\stackrel{d}{d} \\
\hdashline}}{d}$ & ঙ욜 & 놈 & 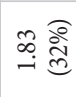 & in & 象高 & 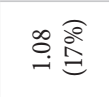 & 징 & 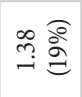 \\
\hline 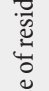 & $\begin{array}{l}\text { small / middle } \\
\text { size town }\end{array}$ & 돌 & 욤 & in & 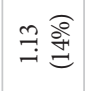 & क्ष & 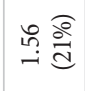 & 㝑 & 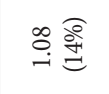 & 늄 & 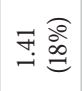 \\
\hline$\frac{\pi}{2}$ & village & 동 & ํㅠㄱ & 花 & 苍 & م્ & 닥 & 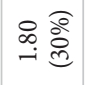 & 능 & 号 高 & ले \\
\hline & $\stackrel{+}{\stackrel{+}{n}}$ & 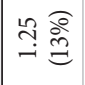 & ت & $\begin{array}{l}0 \\
\infty \\
0 \\
0\end{array}$ & 依 & 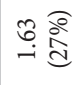 & $\stackrel{\Re}{\stackrel{9}{\stackrel{9}{\ominus}}}$ & in & 点 & 공 & à \\
\hline & $\begin{array}{l}\text { H } \\
5 \\
\text { \& }\end{array}$ & 在 & 足 & 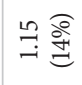 & g̊ & 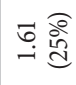 & $\stackrel{n}{\stackrel{0}{\stackrel{d}{d}}}$ & 송 & م̆ & 월 & $\stackrel{\dddot{a}}{=}$ \\
\hline 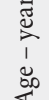 & $\begin{array}{l}\text { J } \\
\text { in } \\
\text { 吕 }\end{array}$ & 蒙 & $\stackrel{\circ}{\stackrel{\circ}{\circ}}$ & 萑 & 국 & 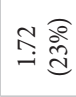 & 엄 & 는 & 울 & 동 & 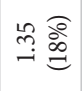 \\
\hline & $\begin{array}{l}\stackrel{+}{w} \\
\stackrel{1}{\sim}\end{array}$ & ळ & 총 & 육 & ‡ & 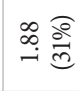 & in & 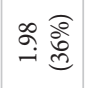 & $\stackrel{2}{\stackrel{9}{i}}$ & 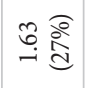 & $\stackrel{\infty}{\stackrel{0}{q}}$ \\
\hline & $\begin{array}{l}\stackrel{1}{1} \\
\stackrel{n}{n}\end{array}$ & 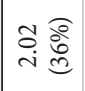 & ๙ & $\stackrel{\infty}{\rightarrow} \underset{\frac{\rho}{c}}{\frac{\rho}{c}}$ & $\stackrel{\overparen{9}}{\rightarrow}$ & 핳 & 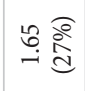 & 虽 & 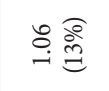 & 㐫 $\overline{\overbrace{}^{\circ}}$ & 둥 \\
\hline 它 & 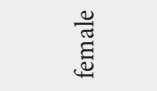 & 공 & 암 & ঙㅇ & 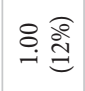 & 동 & 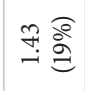 & 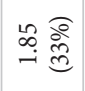 & Ð艹 & $\stackrel{n}{\stackrel{\overbrace{}}{\rightarrow}} \underset{d}{d}$ & ले \\
\hline & $\frac{\ddot{\Xi}}{\tilde{\Xi}}$ & $\stackrel{\infty}{\stackrel{\substack{\stackrel{d}{d} \\
d}}{d}}$ & 엉 & $\stackrel{7}{\rightarrow} \stackrel{\stackrel{\partial}{a}}{\stackrel{d}{d}}$ & 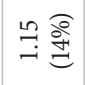 & 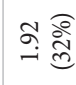 & 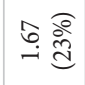 & 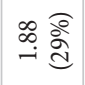 & 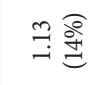 & 임 & F \\
\hline & 吾 & 욤 & $\stackrel{\substack{\stackrel{a}{d} \\
d}}{d}$ & ঙ 일 & 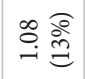 & ô & 负 & 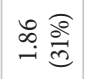 & 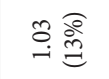 & in & ले \\
\hline & 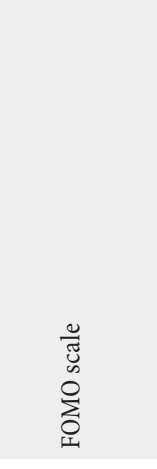 & 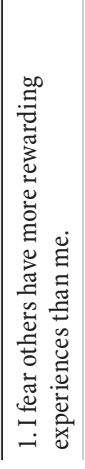 & 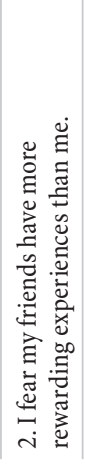 & 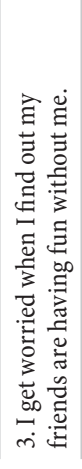 & 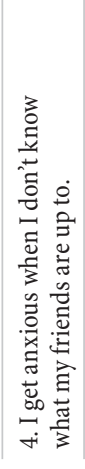 & 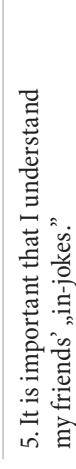 & 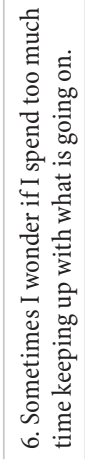 & 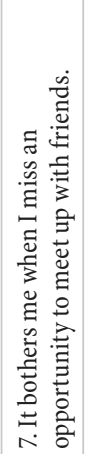 & 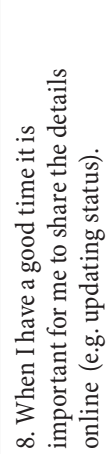 & 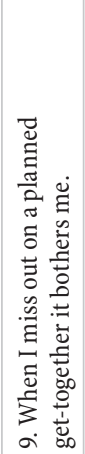 & 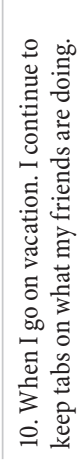 \\
\hline
\end{tabular}

Source: Authors 
Table 3. Statistical significance of the FOMO 10-item battery set - demographic variables $(p<0.05)$

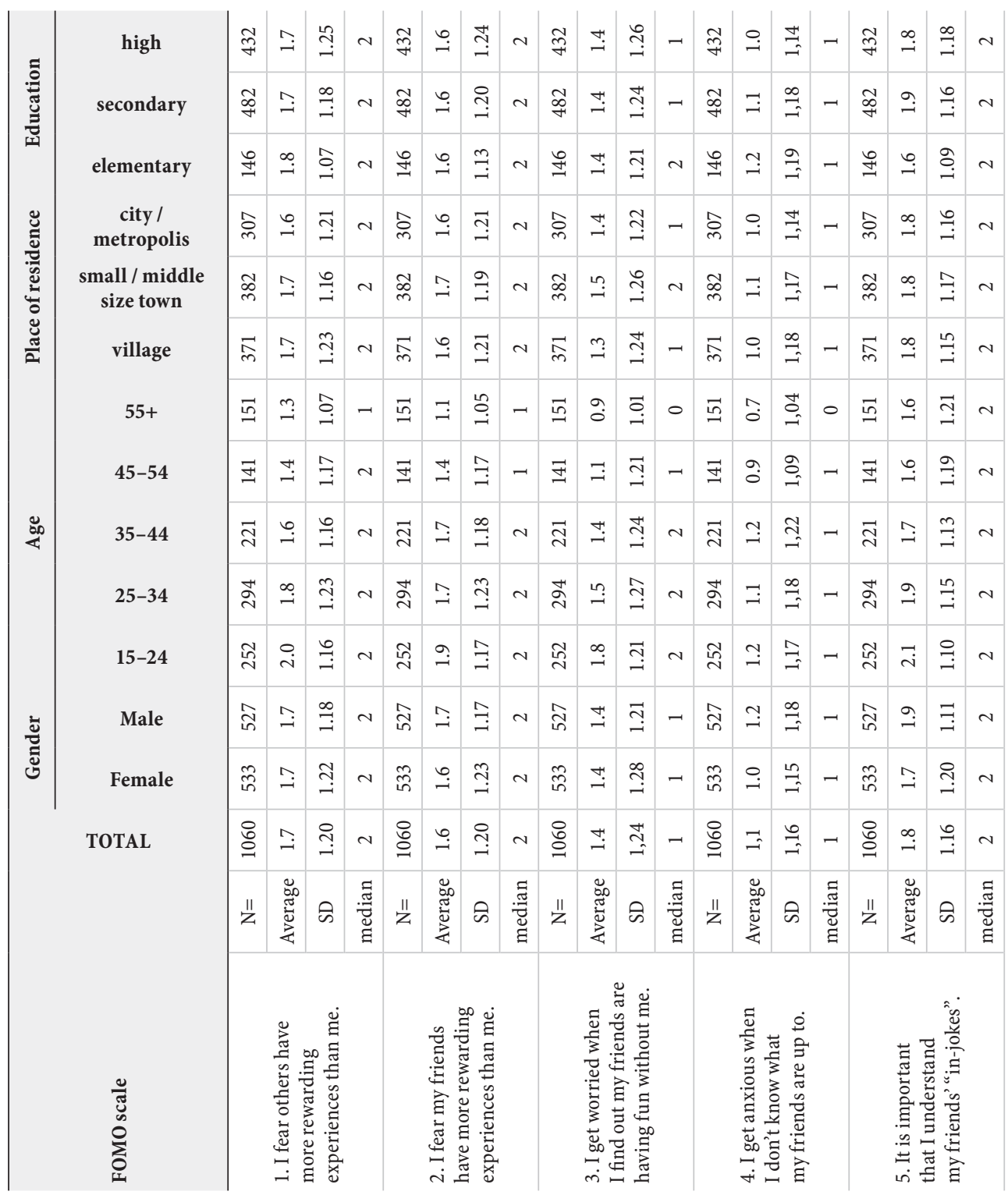




\begin{tabular}{|c|c|c|c|c|c|c|c|c|c|c|c|c|c|c|c|c|c|c|}
\hline & high & $\approx$ & $\stackrel{\text { ?ִ }}{\longrightarrow}$ & ક & 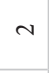 & $\tilde{F}$ & $9 \stackrel{6}{=}$ & $\sim$ & $\tilde{F}$ & $\grave{o}$ & $\stackrel{\varrho}{=}$ & 0 & $\widetilde{F}$ & 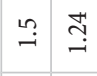 & N & $\approx \stackrel{F}{F}$ & $\stackrel{\infty}{=}$ & 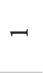 \\
\hline 訰 & secondary & $\stackrel{\mathscr{\infty}}{+}$ & $\stackrel{\circ}{-}$ & $\stackrel{\varrho}{=}$ & $\sim$ & $\underset{\substack{\infty \\
+}}{\infty}$ & $\stackrel{9}{=}$ & N & $\stackrel{\sim}{\infty}$ & $\exists$ & 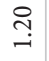 & - & 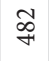 & $\stackrel{0}{-} \vec{I}$ & $\sim$ & $\underset{\stackrel{\infty}{\sim}}{\stackrel{\sim}{+}}$ & $\stackrel{\overbrace{}}{\overbrace{}}$ & - \\
\hline & elementary & I & $\stackrel{6}{-}$ & $\exists$ & $\sim$ & I & 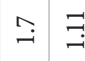 & $\sim$ & I & $\stackrel{\text { Iִ }}{-}$ & $\stackrel{2}{\leftrightarrows}$ & - & $\stackrel{0}{+}$ & 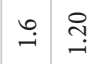 & $\sim$ & 导 & กี & $\sim$ \\
\hline$\ddot{\Xi}$ & $\begin{array}{c}\text { city / } \\
\text { metropolis }\end{array}$ & 今े & $\stackrel{0}{-}$ & $\stackrel{2}{=}$ & $\sim$ & 命 & $\stackrel{\Xi}{=}$ & N & 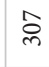 & $=$ & $\vec{\Im}$ & - & 今్ & $\stackrel{\bullet}{\rightarrow} \stackrel{\sim}{\rightarrow}$ & $\sim$ & 今े & $\stackrel{2}{=}$ & 7 \\
\hline$\frac{5}{2}$ & $\begin{array}{l}\text { small / middle } \\
\text { size town }\end{array}$ & $\underset{\infty}{\tilde{m}}$ & $\stackrel{\circ}{-}$ & $\cong$ & $\sim$ & $\underset{ల}{\infty}$ & $\stackrel{9}{-1}$ & $N$ & $\underset{\sim}{\infty}$ & $\exists$ & 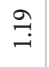 & - & $\underset{ల}{\infty}$ & $\stackrel{\overbrace{}}{-}$ & $\sim$ & $\stackrel{\infty}{\infty}$ & $\stackrel{\infty}{=}$ & $N$ \\
\hline$\frac{\pi}{2}$ & village & 离 & $\stackrel{n}{\rightarrow}$ & $\stackrel{0}{\dddot{1}}$ & $\sim$ & $\vec{n}$ & 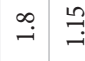 & 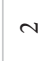 & त & $\grave{0}$ & $\stackrel{m}{=}$ & 0 & 离 & 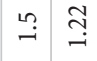 & $\sim$ & 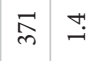 & $\stackrel{\widetilde{T}}{\overbrace{}}$ & - \\
\hline & $55+$ & 도 & $\stackrel{\sharp}{\longrightarrow}$ & $\stackrel{\infty}{\stackrel{\infty}{-}}$ & - & $\vec{n}$ & $\stackrel{\text { L }}{\rightarrow} \stackrel{\varrho}{=}$ & v & 돔 & $\hat{o}$ & $\stackrel{8}{\circ}$ & 0 & $\sqrt{n}$ & $\stackrel{\overbrace{}}{\Im}$ & $\neg$ & $\vec{n} \stackrel{\circ}{-}$ & $\underset{7}{ت}$ & 0 \\
\hline & $45-54$ & $\exists$ & $\stackrel{\sharp}{-}$ & $\stackrel{m}{=}$ & 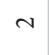 & $\exists$ & $\stackrel{\infty}{\rightarrow} \stackrel{\infty}{=}$ & $\sim$ & $\exists$ & $\grave{0}$ & $\exists$ & 0 & $\exists$ & 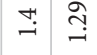 & - & $\exists$ & $\stackrel{\circ}{=}$ & $\neg$ \\
\hline$\stackrel{8}{4}$ & $35-44$ & $\overrightarrow{\text { ส }}$ & $\stackrel{\bullet}{-}$ & 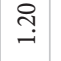 & 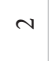 & $\overrightarrow{\widetilde{N}}$ & 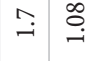 & N & $\overrightarrow{\mathrm{N}}$ & $\stackrel{\text { Tִ }}{-}$ & $\stackrel{\stackrel{2}{\rightarrow}}{\rightarrow}$ & - & $\overrightarrow{\widetilde{N}}$ & $\stackrel{n}{\rightarrow} \stackrel{\text { กิ }}{-}$ & $\sim$ & $\overrightarrow{\mathrm{N}} \stackrel{n}{-}$ & $\stackrel{\vec{\tau}}{-}$ & - \\
\hline & $25-34$ & ন্ & $\stackrel{0}{-}$ & $\stackrel{\sharp}{ت}$ & $N$ & ते & $\stackrel{\circ}{i} \stackrel{\infty}{=}$ & 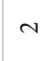 & त) & $\stackrel{\sim}{I}$ & $\stackrel{ت}{ت}$ & - & స్ & $\stackrel{0}{-} \stackrel{\sharp}{\stackrel{I}{I}}$ & $\sim$ & ఫ્ત゙ & ঙิ & 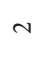 \\
\hline & $15-24$ & กิ & 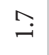 & 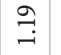 & $\sim$ & กี & $\vec{i} \stackrel{g}{\mathrm{i}}$ & N & กิ & $\exists$ & $\stackrel{20}{=}$ & $\rightarrow$ & กิ & $\stackrel{9}{\rightarrow}$ & $v$ & $\stackrel{\widetilde{N}}{\stackrel{N}{N}} \stackrel{\bullet}{-}$ & $\stackrel{2}{\leftrightarrows}$ & $\sim$ \\
\hline & Male & $\widehat{\overbrace{}}$ & $\cong$ & $\stackrel{\overbrace{}}{-}$ & $\sim$ & $\widehat{\widehat{ก}}$ & 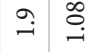 & 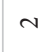 & $\widehat{\hat{n}}$ & $\exists$ & $\stackrel{\vec{\jmath}}{\leftrightarrows}$ & - & $\widehat{\hat{n}}$ & $\stackrel{0}{0} \stackrel{-}{\longrightarrow}$ & $N$ & กิ & ঙิ & N \\
\hline & Female & î & $\stackrel{\rightleftarrows}{-}$ & $\vec{ָ}$ & $\neg$ & กิ & 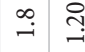 & N & î̉ & $\grave{o}$ & $\stackrel{2}{=}$ & 0 & $\stackrel{\widetilde{R}}{i n}$ & 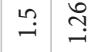 & $\sim$ & ⿵ి & 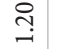 & $\neg$ \\
\hline & TOTAL & $\stackrel{8}{\circ}$ & $\stackrel{\leftrightarrow}{\rightarrow}$ & $\stackrel{0}{=}$ & $\sim$ & $\stackrel{8}{\circ}$ & $\stackrel{I}{=}$ & v & $\stackrel{\circ}{\circ}$ & $\stackrel{\circ}{-}$ & $\stackrel{\infty}{=}$ & - & $\stackrel{8}{\circ}$ & 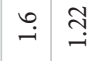 & v & $\stackrel{8}{\circ}$ & $\stackrel{\overbrace{}}{\stackrel{9}{i}}$ & $\neg$ \\
\hline & & " & 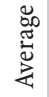 & के & 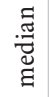 & z & 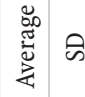 & 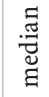 & $\ddot{z}$ & 离 & के & 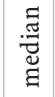 & Z & 旁 & 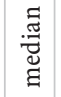 & $\begin{array}{ll}\text { 乙 } \\
\text { 乙 }\end{array}$ & के & 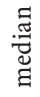 \\
\hline & 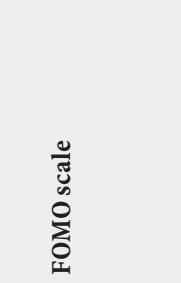 & & 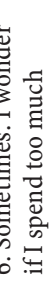 & 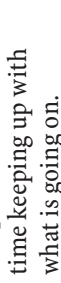 & & & 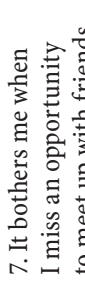 & & & 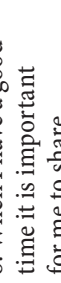 & 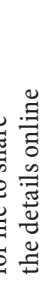 & & & 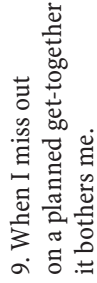 & & 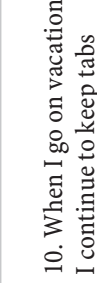 & 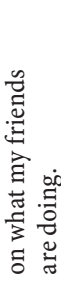 & \\
\hline
\end{tabular}

Then the FOMO scale index was developed, aggregating results for the FOMO scale. The index took the form of recoded (linearly transformed) results of the 10 -statement set to the range of the final value $0-100$. We decided to present the results as an index in the $0-100$ range to make them more understandable and distinct for all recipients. As mentioned, the results from our extended questionnaire, including FOMO scale set, were presented in a special report which was highly commented and cited by Polish media and non-academic media users (Authors, 2018). 
The standard deviation was close to the normal distribution (see: Figure 1), therefore we decided to refer definitions of FOMO levels to it. A high standard deviation $(>1 \sigma)$ indicated 'high FOMO' in range 60-100 on index score, a 'low FOMO' level was based on values $<-1 \sigma$, getting $0-20$ range score, and an average or 'mid FOMO' level was based on a the range of values 21-59.

Recalculation of the FOMO scale results into the FOMO scale index and (finally) in percentages, enabled clear presentation of the range of FOMO phenomenon in the sample population, and potential further comparisons among populations, if similar representative surveys are realised.

All statistical tests were performed using SPSS Statistics version 23.0. The FOMO items showed good internal consistency and reliability Cronbach's $\alpha=$ .89 , complementing previous international studies. The FOMO index range scores among primary measures variables, including means and standard deviations, are presented in Figure 1.

Figure 1. The FOMO index range scores

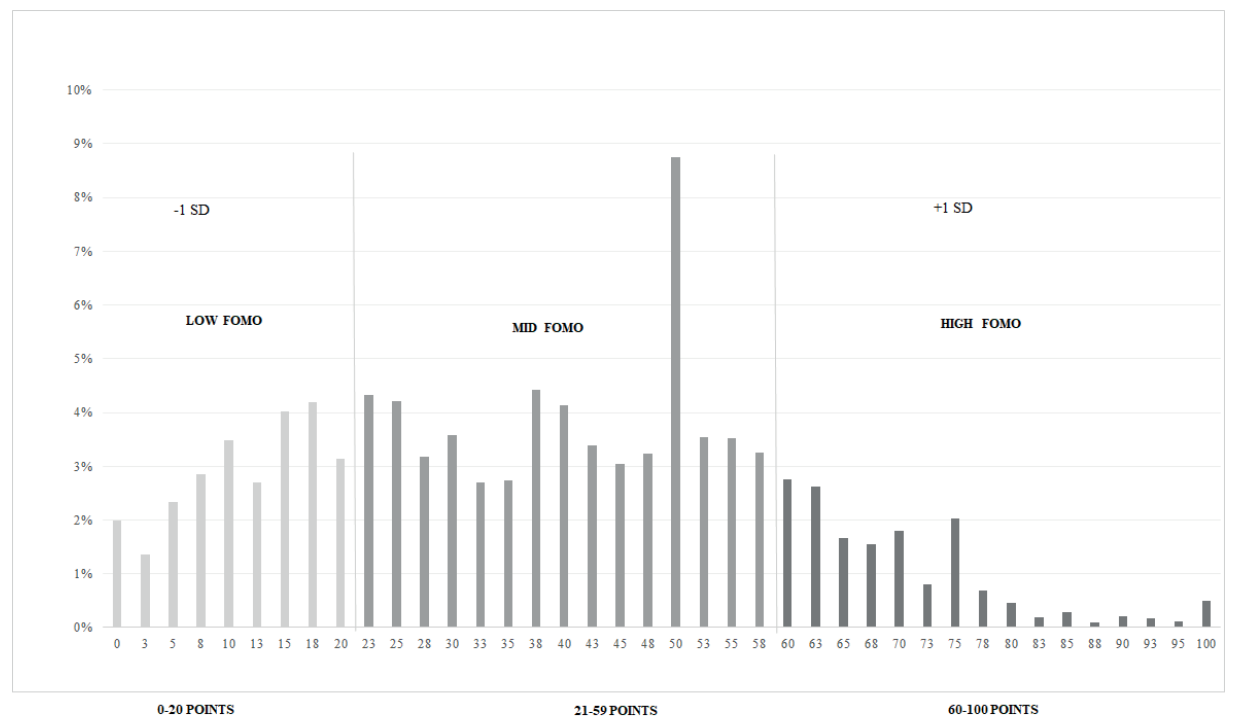

Source: Authors 


\section{RESULTS AND DISCUSSION}

\section{FOMO OCCURRENCE IN POLAND - DEMOGRAPHIC VARIABLES}

Detailed data on the FOMO occurrence among Poland's internet users are presented in Table 4.

Table 4. Range of FOMO occurrence - demographic variables (\%)

\begin{tabular}{|c|c|c|c|}
\hline Sample variables $\backslash$ FOMO scale & Low FOMO & Mid FOMO & High FOMO \\
\hline Total & 19 & 65 & 16 \\
\hline \multicolumn{4}{|c|}{ Gender } \\
\hline male & 18 & 67 & 15 \\
\hline female & 19 & 64 & 17 \\
\hline \multicolumn{4}{|c|}{ Age-years } \\
\hline $15-24$ & 10 & 69 & 21 \\
\hline $25-34$ & 14 & 69 & 17 \\
\hline $35-44$ & 21 & 61 & 18 \\
\hline $45-54$ & 27 & 61 & 12 \\
\hline $55+$ & 2 & 65 & 6 \\
\hline \multicolumn{4}{|c|}{ Place of residence } \\
\hline village & 18 & 68 & 14 \\
\hline small and middle size town & 19 & 65 & 16 \\
\hline city and metropolis & 18 & 64 & 18 \\
\hline \multicolumn{4}{|c|}{ Education level } \\
\hline primary & 19 & 66 & 15 \\
\hline secondary & 18 & 66 & 16 \\
\hline high & 19 & 65 & 16 \\
\hline
\end{tabular}

As mentioned above, we identified three categories of the Poland's 'FOMOpeople', depending on their FOMO characteristics. The higher FOMO indicator refers to $16 \%$ of the 'high FOMO' respondents. The larger is the 'mid FOMO' group of the users (65\%), and the 'low FOMO' includes 19\% of them.

As for the age characteristics, approximately $21 \%$ of the youngest population (aged $15-24$ years), $17 \%$ of the next (25-34 years), and $18 \%$ the following (35-44 years) suffer from high FOMO. The low FOMO attains the highest value $(30 \%)$ in the group $55+$ years. 
Significant differences were not found between men (15\% of them are 'high FOMO') and women (17\% 'high FOMO'). The same can be observed for the place of living ( $4 \%$ of difference between rural areas and big cities in the "high FOMO' group, no differences among the so-called 'low FOMO'), and education.

\section{THE FOMO SCALE IN POLAND}

The analysis of the statistical significance of the range of the phenomenon's occurrence in the context of the FOMO scale are presented in Table $5(\mathrm{p}<0.05)$. As previously mentioned, light grey marking means that the subgroup in question has a significantly higher response value (and a dark grey marking means that the subgroup in question has a significantly lower response value).

Table 5. Statistical significance of the range of the FOMO phenomenon's occurrence in the context of the FOMO scale $(\mathbf{p}<0.05)$

\begin{tabular}{|c|c|c|c|c|c|}
\hline FOMO scale & & Total & low FOMO & mid FOMO & $\begin{array}{l}\text { high } \\
\text { FOMO }\end{array}$ \\
\hline \multirow{4}{*}{$\begin{array}{l}\text { 1. I fear others have more rewarding } \\
\text { experiences than me. }\end{array}$} & $\mathrm{N}=$ & 1060 & 196 & 695 & 169 \\
\hline & Average & 1.7 & 0.5 & 1.7 & 2.9 \\
\hline & $\mathrm{SD}$ & 1.20 & 0.76 & 1.05 & 0.83 \\
\hline & median & 2 & 0 & 2 & 3 \\
\hline \multirow{4}{*}{$\begin{array}{l}\text { 2. I fear my friends have more } \\
\text { rewarding experiences than me. }\end{array}$} & $\mathrm{N}=$ & 1060 & 196 & 695 & 169 \\
\hline & Average & 1.6 & 0.4 & 1.7 & 2.8 \\
\hline & $\mathrm{SD}$ & 1.20 & 0.70 & 1.05 & 0.88 \\
\hline & median & 2 & 0 & 2 & 3 \\
\hline \multirow{4}{*}{$\begin{array}{l}\text { 3. I get worried when I find out } \\
\text { my friends are having fun without me. }\end{array}$} & $\mathrm{N}=$ & 1060 & 196 & 695 & 169 \\
\hline & Average & 1.4 & 0.2 & 1.4 & 2.9 \\
\hline & $\mathrm{SD}$ & 1.24 & 0.49 & 1.07 & 0.83 \\
\hline & median & 1 & 0 & 2 & 3 \\
\hline \multirow{4}{*}{$\begin{array}{l}\text { 4. I get anxious when I don't know } \\
\text { what my friends are up to. }\end{array}$} & $\mathrm{N}=$ & 1060 & 196 & 695 & 169 \\
\hline & Average & 1.1 & 0.1 & 1.0 & 2.5 \\
\hline & $\mathrm{SD}$ & 1.16 & 0.31 & 1.03 & 0.99 \\
\hline & median & 1 & 0 & 1 & 3 \\
\hline \multirow{4}{*}{$\begin{array}{l}\text { 5. It is important that I understand } \\
\text { my friends' "in-jokes". }\end{array}$} & $\mathrm{N}=$ & 1060 & 196 & 695 & 169 \\
\hline & Average & 1.8 & 0.6 & 1.9 & 2.9 \\
\hline & SD & 1.16 & 0.82 & 1.00 & 0.68 \\
\hline & median & 2 & 0 & 2 & 3 \\
\hline
\end{tabular}




\begin{tabular}{|c|c|c|c|c|c|}
\hline FOMO scale & & Total & low FOMO & mid FOMO & $\begin{array}{c}\text { high } \\
\text { FOMO }\end{array}$ \\
\hline \multirow{4}{*}{$\begin{array}{l}\text { 6. Sometimes, I wonder } \\
\text { if I spend too much time keeping } \\
\text { up with what is going on. }\end{array}$} & $\mathrm{N}=$ & 1060 & 196 & 695 & 169 \\
\hline & Average & 1.5 & 0.4 & 1.6 & 2.8 \\
\hline & $\mathrm{SD}$ & 1.16 & 0.72 & 1.02 & 0.82 \\
\hline & median & 2 & 0 & 2 & 3 \\
\hline \multirow{4}{*}{$\begin{array}{l}\text { 7. It bothers me when I miss } \\
\text { an opportunity to meet up with friends. }\end{array}$} & $\mathrm{N}=$ & 1060 & 196 & 695 & 169 \\
\hline & Average & 1.9 & 0.7 & 1.9 & 3.0 \\
\hline & $\mathrm{SD}$ & 1.14 & 0.77 & 0.98 & 0.75 \\
\hline & median & 2 & 0 & 2 & 3 \\
\hline \multirow{4}{*}{$\begin{array}{l}\text { 8. When I have a good time } \\
\text { it is important for me to share the } \\
\text { details online (e.g. updating status). }\end{array}$} & $\mathrm{N}=$ & 1060 & 196 & 695 & 169 \\
\hline & Average & 1.0 & 0.1 & 1.0 & 2.4 \\
\hline & $\mathrm{SD}$ & 1.18 & 0.39 & 1.05 & 1.11 \\
\hline & median & 1 & 0 & 1 & 3 \\
\hline \multirow{4}{*}{$\begin{array}{l}\text { 9. When I miss out on a planned } \\
\text { get-together it bothers me. }\end{array}$} & $\mathrm{N}=$ & 1060 & 196 & 695 & 169 \\
\hline & Average & 1.6 & 0.3 & 1.6 & 3.0 \\
\hline & $\mathrm{SD}$ & 1.22 & 0.59 & 1.06 & 0.78 \\
\hline & median & 2 & 0 & 2 & 3 \\
\hline \multirow{4}{*}{$\begin{array}{l}\text { 10. When I go on vacation, I continue } \\
\text { to keep tabs on what my friends are doing. }\end{array}$} & $\mathrm{N}=$ & 1060 & 196 & 695 & 169 \\
\hline & Average & 1.4 & 0.3 & 1.4 & 2.8 \\
\hline & $\mathrm{SD}$ & 1.20 & 0.58 & 1.03 & 0.93 \\
\hline & median & 1 & 0 & 2 & 3 \\
\hline
\end{tabular}

Source: Authors

For data on the range of the FOMO occurrence in the context of the FOMO scale (see Table 6). Due to the huge quantity of data, combined results for indications of 'true about me' and 'extremely true about me' are presented.

Table 6. The range of FOMO occurrence - the FOMO scale

\begin{tabular}{|c|c|c|c|c|}
\hline FOMO scale & $\begin{array}{l}\text { Total number } \\
\text { of respondents }\end{array}$ & Low FOMO & Mid FOMO & $\begin{array}{l}\text { High } \\
\text { FOMO }\end{array}$ \\
\hline $\begin{array}{l}\text { 1. I fear others have more rewarding } \\
\text { experiences than me. }\end{array}$ & $1.70(26 \%)$ & $0.52(2 \%)$ & $1.74(20 \%)$ & $2.91(74 \%)$ \\
\hline $\begin{array}{l}\text { 2. I fear my friends have more } \\
\text { rewarding experiences than me. }\end{array}$ & $1.63(24 \%)$ & $0.39(2 \%)$ & $1.69(29 \%)$ & $2.81(69 \%)$ \\
\hline $\begin{array}{l}\text { 3. I get worried when I find out my friends } \\
\text { are having fun without me. }\end{array}$ & $1.43(22 \%)$ & $0.21(0 \%)$ & $1.40(14 \%)$ & $2.94(80 \%)$ \\
\hline $\begin{array}{l}\text { 4. I get anxious when I don't know } \\
\text { what my friends are up to. }\end{array}$ & $1.08(13 \%)$ & $0.09(0 \%)$ & $1.02(7 \%)$ & $2.47(55 \%)$ \\
\hline
\end{tabular}




\begin{tabular}{|c|c|c|c|c|}
\hline FOMO scale & $\begin{array}{l}\text { Total number } \\
\text { of respondents }\end{array}$ & Low FOMO & Mid FOMO & $\begin{array}{l}\text { High } \\
\text { FOMO }\end{array}$ \\
\hline $\begin{array}{l}\text { 5. It is important that I understand } \\
\text { my friends' „in jokes.” }\end{array}$ & $1.82(30 \%)$ & $0.58(3 \%)$ & $1.90(27 \%)$ & $2.94(76 \%)$ \\
\hline $\begin{array}{l}\text { 6. Sometimes I wonder if I spend too much } \\
\text { time keeping up with what is going on. }\end{array}$ & $1.55(21 \%)$ & $0.45(1 \%)$ & $1.57(15 \%)$ & $2.75(69 \%)$ \\
\hline $\begin{array}{l}\text { 7. It bothers me when I miss an opportunity } \\
\text { to meet up with friends. }\end{array}$ & $1.86(31 \%)$ & $0.65(1 \%)$ & $1.93(28 \%)$ & $2.98(78 \%)$ \\
\hline $\begin{array}{l}\text { 8. When I have a good time } \\
\text { it is important for me to share the } \\
\text { details online (e.g. updating status). }\end{array}$ & $1.03(13 \%)$ & $0.11(1 \%)$ & $0.96(7 \%)$ & $2.38(55 \%)$ \\
\hline $\begin{array}{l}\text { 9. When I miss out on a planned } \\
\text { get-together it bothers me. }\end{array}$ & $1.57(24 \%)$ & $0.30(1 \%)$ & $1.60(18 \%)$ & $2.95(77 \%)$ \\
\hline $\begin{array}{l}\text { 10. When I go on vacation. I continue to keep } \\
\text { tabs on what my friends are doing. }\end{array}$ & $1.39(19 \%)$ & $0.27(1 \%)$ & $1.37(12 \%)$ & $2.77(68 \%)$ \\
\hline means at $0-4$ scale (\% top2box) & & & & \\
\hline
\end{tabular}

Source: Authors

The answers declared within the FOMO scale differ from the answers analysed in the general approach, i.e. averaged for the whole sample. They are the most similar for the 'mid FOMO' people, and the least for the 'high FOMO' people. The reference group of people who have more 'rewarding experiences' seems to be important for the respondents, and is more often encountered in the real life than the virtual world. The fear of others having a more interesting life than the respondents themselves is troublesome, as are missed meetings. Activities on social media have not gained a strong position here - although it is true that during holidays respondents check their friends' profiles, but when they have fun, they do not update their own profiles very often.

The answers for mid-FOMO people are quite similar to the total sample, although with some exceptions. First, they declare compliance with the claims from the FOMO scale less often than the total sample. The exception applies to statement 2, which at the same time received the most indications (29\%). This may prove the importance of a reference group (especially friends), which clearly strengthens the feeling of fear in the context of the assessment of one's own, satisfying experiences.

In the case of people with low FOMO, all results are between $0-3 \%$, and statements 5,1 and 2 can be regarded as the most emotional ones.

People with high FOMO clearly 'stand out' from the other groups: first, because of the scale of affirmative answers and, secondly, because of the slightly different order of statements compared to the total sample and mid FOMO group. The reference group is very important for this high FOMO group of people: mainly 
how and with whom they spend time. The respondents are clearly anxious about their absence at meetings, and more so when it refers to events they did not know about. Acquiring satisfying experiences remains crucial, but the influence of 'other people' (not only friends) is slightly more important here. Social media are also in the middle and at the end of the list, but it is worth noting that their significance - compared to other groups of respondents - increased very noticeably.

\section{DEMOGRAPHIC CHARACTERISTICS OF THE 'HIGH FOMO” PEOPLE}

This subgroup, analysed in terms of gender (see Table 7), is characterised by a high uniformity of responses. In most cases, women express their fears a little more often. This pattern is evident in the answers of six (out of 10) statements. In turn, men are more afraid of missing a meeting with friends $(+5 \%)$, they are more interested in boasting on the internet about what they are doing $(+12 \%)$ or more concerned when they do not know what their friends are doing $(+6 \%)$. At the same time, they wonder if they spend too much time in the virtual world more often than women $(+14 \%$, statement 6$)$.

Table 7. Demographic characteristics of high FOMO phenomenon - gender (\%)

\begin{tabular}{|c|c|c|c|}
\hline \multirow{2}{*}{ FOMO scale } & \multicolumn{3}{|c|}{ High FOMO People } \\
\hline & Total & Women & Men \\
\hline $\begin{array}{l}\text { 1. I fear others have more rewarding } \\
\text { experiences than me. }\end{array}$ & 74.4 & 79.5 & 68.7 \\
\hline $\begin{array}{l}\text { 2. I fear my friends have more } \\
\text { rewarding experiences than me. }\end{array}$ & 69.4 & 77.1 & 60.8 \\
\hline $\begin{array}{l}\text { 3. I get worried when I find out my friends } \\
\text { are having fun without me. }\end{array}$ & 80.0 & 84.2 & 75.3 \\
\hline $\begin{array}{l}\text { 4. I get anxious when I don't know } \\
\text { what my friends are up to. }\end{array}$ & 55.0 & 52.1 & 58.3 \\
\hline $\begin{array}{l}\text { 5. It is important that I understand } \\
\text { my friends" "in-jokes". }\end{array}$ & 76.3 & 76.4 & 76.1 \\
\hline $\begin{array}{l}\text { 6. Sometimes, I wonder if I spend too much } \\
\text { time keeping up with what is going on. }\end{array}$ & 69.4 & 62.5 & 77.0 \\
\hline $\begin{array}{l}\text { 7. It bothers me when I miss an opportunity } \\
\text { to meet up with friends. }\end{array}$ & 77.8 & 81.9 & 73.3 \\
\hline $\begin{array}{l}\text { 8. When I have a good time it is important for } \\
\text { me to share the details online (e.g. updating status). }\end{array}$ & 54.5 & 48.4 & 61.4 \\
\hline $\begin{array}{l}\text { 9. When I miss out on a planned } \\
\text { get-together it bothers me. }\end{array}$ & 76.5 & 73.7 & 79.5 \\
\hline $\begin{array}{l}\text { 10. When I go on vacation, I continue to keep } \\
\text { tabs on what my friends are doing. }\end{array}$ & 68.0 & 69.0 & 66.9 \\
\hline
\end{tabular}

Source: Authors 
In the case of the second variable of age (see Table 8), the data for respondents aged 55 years or more are quite unambiguous. The rate of answers confirming the occurrence of fear is definitely higher than the average for the whole high FOMO group (this applies to six statements). This may indicate a greater awareness during the answering process or a greater involvement in virtual life (for example all respondents aged 55 years and more agreed with statement 10). This result may be related to internet addiction, manifested in compulsive checking of what is happening in the virtual world, as well as attaching great importance to online life. The lowest indicators were noted for the age group 45-55 years, while the youngest respondents (15-24 years) pointed out the loss of the opportunity to meet friends and the fear that friends experienced more than they did themselves (just like the age group 55 years or more). The youngest and oldest respondents more often emphasized the importance of humour: this indicates the importance of identification with a given reference group and the need to belong to it.

Table 8. Demographic characteristic - age (\%) - of high FOMO people - age (\%)

\begin{tabular}{|c|c|c|c|c|c|c|}
\hline \multirow[b]{2}{*}{ FOMO scale } & \multicolumn{6}{|c|}{ High FOMO People } \\
\hline & Total & $\begin{array}{l}15-24 \\
\text { years }\end{array}$ & $\begin{array}{l}25-34 \\
\text { years }\end{array}$ & $\begin{array}{l}35-44 \\
\text { years }\end{array}$ & $\begin{array}{l}45-54 \\
\text { years }\end{array}$ & $\begin{array}{l}55 \text { years } \\
\text { or older }\end{array}$ \\
\hline $\begin{array}{l}\text { 1. I fear others have more } \\
\text { rewarding experiences than me. }\end{array}$ & 74.4 & 73.5 & 82.6 & 66.5 & 67.3 & 80.2 \\
\hline $\begin{array}{l}\text { 2. I fear my friends have more } \\
\text { rewarding experiences than me. }\end{array}$ & 69.4 & 77.6 & 67.7 & 66.4 & 49.6 & 83.8 \\
\hline $\begin{array}{l}\text { 3. I get worried when I find out } \\
\text { my friends are having fun without me. }\end{array}$ & 80.0 & 73.0 & 86.0 & 85.0 & 83.5 & 55.9 \\
\hline $\begin{array}{l}\text { 4. I get anxious when I don't know } \\
\text { what my friends are up to. }\end{array}$ & 55.0 & 54.4 & 47.6 & 69.2 & 50.1 & 48.6 \\
\hline $\begin{array}{l}\text { 5. It is important that I understand } \\
\text { my friends' "in jokes". }\end{array}$ & 76.3 & 82.2 & 75.0 & 70.0 & 72.9 & 83.8 \\
\hline $\begin{array}{l}\text { 6. Sometimes, I wonder } \\
\text { if I spend too much time keeping } \\
\text { up with what is going on. }\end{array}$ & 69.4 & 67.5 & 65.6 & 77.1 & 61.4 & 83.5 \\
\hline $\begin{array}{l}\text { 7. It bothers me when I miss } \\
\text { an opportunity to meet } \\
\text { up with friends. }\end{array}$ & 77.8 & 82.4 & 80.3 & 76.6 & 56.4 & 83.8 \\
\hline $\begin{array}{l}\text { 8. When I have a good time } \\
\text { it is important for me to share the } \\
\text { details online (e.g. updating status). }\end{array}$ & 54.5 & 46.1 & 53.4 & 66.5 & 54.7 & 55.9 \\
\hline $\begin{array}{l}\text { 9. When I miss out on a planned } \\
\text { get-together it bothers me. }\end{array}$ & 76.5 & 74.7 & 80.7 & 77.1 & 67.9 & 75.7 \\
\hline $\begin{array}{l}\text { 10. When I go on vacation, } \\
\text { I continue to keep tabs on what } \\
\text { my friends are doing. }\end{array}$ & 68.0 & 65.2 & 70.8 & 71.4 & 44.3 & 100.0 \\
\hline
\end{tabular}

Source: Authors 
Regarding the place of residence (see Table 9), the results for all subgroups are very similar. The average of positive responses for the entire high FOMO group to specific questions is the same as for the residents of the largest urban areas. The results for residents of small and medium-sized towns were on average $1 \%$ greater than those resident in cities (71.3\% against $70.1 \%)$, while the results for residents of rural areas were $1 \%$ lower (68.8\%). The latter high FOMO people are mainly residents of small and medium-sized rural towns who are afraid that they will miss an event organized by, or gain less experience than, their friends. It is especially important for them to know the jokes characteristic for a given group of friends (87.1\%, which is the highest score among all fears) - which indicates the need for acceptance and identification.

Table 9. Demographic characteristic - place of residence (\%) - of high FOMO people

\begin{tabular}{|c|c|c|c|c|}
\hline \multirow[b]{2}{*}{ FOMO scale } & \multicolumn{4}{|c|}{ High FOMO People } \\
\hline & Total & villages & $\begin{array}{c}\text { Small } \\
\text { or middle } \\
\text { size town }\end{array}$ & $\begin{array}{l}\text { City and } \\
\text { metropolis }\end{array}$ \\
\hline $\begin{array}{l}\text { 1. I fear others have more } \\
\text { rewarding experiences than me. }\end{array}$ & 74.4 & 79.3 & 77.1 & 66.7 \\
\hline $\begin{array}{l}\text { 2. I fear my friends have more } \\
\text { rewarding experiences than me. }\end{array}$ & 69.4 & 73.8 & 69.0 & 65.5 \\
\hline $\begin{array}{l}\text { 3. I get worried when I find out } \\
\text { my friends are having fun without me. }\end{array}$ & 80.0 & 69.0 & 83.1 & 87.0 \\
\hline $\begin{array}{l}\text { 4. I get anxious when I don't know } \\
\text { what my friends are up to. }\end{array}$ & 55.0 & 59.2 & 56.9 & 49.1 \\
\hline $\begin{array}{l}\text { 5. It is important that I understand } \\
\text { my friends' "in-jokes". }\end{array}$ & 76.3 & 67.5 & 87.1 & 72.8 \\
\hline $\begin{array}{l}\text { 6. Sometimes, I wonder } \\
\text { if I spend too much time keeping } \\
\text { up with what is going on. }\end{array}$ & 69.4 & 61.0 & 71.3 & 75.3 \\
\hline $\begin{array}{l}\text { 7. It bothers me when I miss } \\
\text { an opportunity to meet } \\
\text { up with friends. }\end{array}$ & 77.8 & 76.7 & 74.7 & 82.1 \\
\hline $\begin{array}{l}\text { 8. When I have a good time } \\
\text { it is important for me to share the } \\
\text { details online (e.g. updating status). }\end{array}$ & 54.5 & 57.2 & 51.7 & 55.1 \\
\hline $\begin{array}{l}\text { 9. When I miss out on a planned } \\
\text { get-together it bothers me. }\end{array}$ & 76.5 & 71.8 & 80.4 & 76.7 \\
\hline $\begin{array}{l}\text { 10. When I go on vacation, } \\
\text { I continue to keep tabs on what } \\
\text { my friends are doing. }\end{array}$ & 68.0 & 72.1 & 62.0 & 70.6 \\
\hline
\end{tabular}

Source: Authors

While examining the level of education of high FOMO respondents (see Table 10), an increase in positive responses can be observed along with an increase in the 
level of education (see Average Scores) starting with 66\% for primary, $70 \%$ for medium, and up to $71 \%$ for higher education. Higher educated people in 6 out of 10 questions exceed average values score for all respondents from the high FOMO group. It is important for them to identify with a social group (in the context of a sense of humour). They are concerned mostly about the possibility of missing a meeting with friends, and the fear that friends are having a better time at a given moment. The same two feelings dominate among respondents with secondary education. In turn, respondents with primary education are the most afraid that other people have more satisfying / enjoyable experiences $(82 \%)$. Detailed results regarding education in the context of high FOMO are presented in Table 10.

Table 10. Demographic characteristics of high FOMO phenomenon - education (\%)

\begin{tabular}{|c|c|c|c|c|}
\hline \multirow{2}{*}{ FOMO scale } & \multicolumn{4}{|c|}{ High FOMO People } \\
\hline & Total & primary & secondary & higher \\
\hline 1. I fear others have more rewarding experiences than me. & 74.4 & 82.6 & 70.6 & 75.8 \\
\hline $\begin{array}{l}\text { 2. I fear my friends have more } \\
\text { rewarding experiences than me. }\end{array}$ & 69.4 & 72.2 & 67.1 & 71.0 \\
\hline $\begin{array}{l}\text { 3. I get worried when I find out my friends } \\
\text { are having fun without me. }\end{array}$ & 80.0 & 71.4 & 82.0 & 80.5 \\
\hline $\begin{array}{l}\text { 4. I get anxious when I don't know } \\
\text { what my friends are up to. }\end{array}$ & 55.0 & 53.7 & 53.8 & 56.8 \\
\hline 5. It is important that I understand my friends" "in jokes". & 76.3 & 63.3 & 75.0 & 81.7 \\
\hline $\begin{array}{l}\text { 6. Sometimes, I wonder if I spend too much } \\
\text { time keeping up with what is going on. }\end{array}$ & 69.4 & 69.1 & 72.9 & 65.6 \\
\hline $\begin{array}{l}\text { 7. It bothers me when I miss an opportunity } \\
\text { to meet up with friends. }\end{array}$ & 77.8 & 59.3 & 80.0 & 81.3 \\
\hline $\begin{array}{l}\text { 8. When I have a good time it is important for } \\
\text { me to share the details online (e.g. updating status). }\end{array}$ & 54.5 & 52.6 & 50.6 & 59.4 \\
\hline $\begin{array}{l}\text { 9. When I miss out on a planned } \\
\text { get-together it bothers me. }\end{array}$ & 76.5 & 64.6 & 76.6 & 80.1 \\
\hline $\begin{array}{l}\text { 10. When I go on vacation, I continue to keep } \\
\text { tabs on what my friends are doing. }\end{array}$ & 68.0 & 76.8 & 71.9 & 60.9 \\
\hline Total of demographic characteristic scores & 701.30 & 665.60 & 700.50 & 713.10 \\
\hline Average score & 70.13 & 66.56 & 70.05 & 71.31 \\
\hline
\end{tabular}

Source: Authors 


\section{CONCLUSIONS}

FOMO affects all the respondents, although to varying degrees. Accordingly, the present study split the respondents into three groups: high FOMO, mid FOMO, and low FOMO, and described the key demographic variables for each group. The most differentiating variable is age, and the least are place of residence and level of education. Obviously, the incidence of FOMO comes along with style of living and intensity of ICT usage. Belongingness and relationships with peers are also confirmed as important and related to immersion in social media life. Importantly, the older respondents ( 55 years and older) in the high FOMO group gained high scores on the FOMO scale.

We offered the original method of recalculation of the FOMO scale results from figures to percentage data, to develop the FOMO scale index. Consequently, the phenomenon has become countable enabling us to estimate how many people suffer from FOMO. The present study adds a vital element to FOMO related literature, as this seems to be the first study in the world that describes and analyses the FOMO phenomenon in percentage terms representative for internet users from one national population (dated on 2018). The data conversion process can be easily applied in any representative FOMO survey.

The results of this study support other representative surveys (Hampton et al., 2016; Reinecke et al., 2017; Bright \& Logan, 2018) in the aspect of presenting FOMO as one of the inherent features of social media users, increasing their stress and discomfort. Just like in other surveys, the need for belongingness is very important, especially for the youngest respondents, and results in permanent compulsory checking of social media feeds. Nationwide representative data enables further comparative projects with other research of that kind, if realised in the future.

\section{LIMITATIONS AND FUTURE RESEARCH}

The survey was based on declarative data, it did not explore the motives or effects of social media usage. Therefore, the qualitative study concerning these issues should be carried out to complete a picture of reasons and consequences of the high FOMO people's behaviour.

We also believe that a global perspective is required for the topic, to get a perspective of the FOMO phenomenon in various populations or groups with specific demographic characteristics (e.g. adolescents or young adults). Therefore, more representative studies on country populations would be of great value. If they are based on the data recalculation process proposed in this article, it is possible to conduct extensive international comparative analyses, which in turn would help to get knowledge about the scale of FOMO in either or both developed and developing countries and its internet and social networking sites dependencies. 
That would be a factor in development or improvement of digital literacy and digital well-being policies at global and regional levels.

\section{ACKNOWLEDGEMENTS}

The survey in 2018 was conducted by the Ariadna panel. Ariadna combines software used in planning and carrying out consumer and experimental surveys with a Poland-wide research panel of 110000 registered users (aged 15 years and older). Respondents with verified identities take part in all research projects conducted via the panel. The identities of all participants of the panel are validated, but in return all respondents on the panel are anonymous and their personal details remain confidential in line with ECC/ ESOMAR codes of conduct and with Polish data protection laws. The Ariadna panel is PJKPA-quality certified and this quality certificate is awarded following a comprehensive audit by Organizacja Firm Badania Opinii i Rynku (www.ofbor.pl, Polish Association of Public Opinion and Marketing Research Firms). OFBOR is a key organization that operates in the area of public opinion and marketing research in Poland. The publication of the 2018 report on FOMO in Poland was supported by Google Poland.

\section{REFERENCES}

Aarestad, S.H., and Eide, T.A. (2017). Smartphone Restriction and its Effect on Subjective Withdrawal Related Scores. Master's thesis, the University of Bergen, Norway.

Alt, D. (2015). College students' academic motivation, media engagement and fear of missing out. Computers in Human Behavior, 49, 111-119.

Alt, D. (2017). Students' social media engagement and fear of missing out (FoMO) in a diverse classroom. Journal of Computing in Higher Education, 29(2), 388-410.

Auverset, L., Billings, A., and Conlin, L. (2016). Time-shifting vs. appointment viewing: the role of fear of missing out within TV consumption behaviors. Communication \& Society, 29(4), 151-164.

Bailey, A.A., Bonifield, C.M., and Arias, A. (2018). Social media use by young Latin American consumers: An exploration. Journal of Retailing and Consumer Services, 43, 10-19.

Bright, L.F., Logan, K. (2018). Is my fear of missing out (FOMO) causing fatigue? Advertising, social media fatigue, and the implications for consumers and brands. Internet Research, 28(5), 1213-1227.

Casale, S., Rugai, L., and Fioravanti, G. (2018). Exploring the role of positive metacognitions in explaining the association between the fear of missing out and social media addiction. Addictive Behaviors 85: 83-87.

Chotpitayasunondh, V., Douandglas, K.M. (2016). How "phubbing" becomes the norm: The antecedents and consequences of snubbing via smartphone. Computers in Human Behavior, 63, 9-18.

Dhir, A., Yossatorn, Y., Kaur, P., and Chen, S. (2018). Online social media fatigue and psychological wellbeing-A study of compulsive use, fear of missing out, fatigue, anxiety and depression. International Journal of Information Management, 40, 141-152. 
Elhai, J.D., Levine, J.C., Dvorak, R.D., and Hall, B.J. (2016). Fear of missing out, need for touch, anxiety and depression are related to problematic smartphone use. Computers in Human Behavior, 63, 509-516.

Franchina, V., Vanden Abeele, M., van Rooij, A., Lo Coco, G., and De Marez, L. (2018). Fear of Missing Out as a Predictor of Problematic Social Media Use and Phubbing Behavior among Flemish Adolescents. International Journal of Environmental Research and Public Health, 15(10), 243-260.

Fuster, H., Chamarro, A., and Oberst, U. (2017). Fear of missing out, online social networking and mobile phone addiction: A latent profile approach. Aloma: Revista de Psicologia, Ciències de l'Educació i de l'Esport, 35(1), 23-30.

Hampton, K.N., Lu, W., and Shin, I. (2016). Digital media and stress: the cost of caring 2.0. Information, Communication \& Society, 19(9), 1267-1286.

Hetz, P.R., Dawson, C.L., and Cullen, T.A. (2015). Social media use and the fear of missing out (FoMO) while studying abroad. Journal of Research on Technology in Education, 47(4), 259-272.

James, T.L., Lowry, P.B., Wallace, L., and Warkentin, M. (2017). The effect of belongingness on obsessive-compulsive disorder in the use of online social networks. Journal of Management Information Systems, 34(2), 560-596.

Jupowicz-Ginalska, A., Jasiewicz, J., Kisilowska, M., Baran, T., and Wysocki, A. (2018). FOMO. Polacy a lęk przed odłączeniem. Warszawa: WDIB UW. Available at: https://www.wdib.uw.edu.pl/attachments/ article/1992/FOMO.\%20Polacy\%20a\%20l\%C4\%99k\%20przed\%20od\%C5\%82\%C4\%85czeniem\%20 -\%20raport\%20z\%20bada\%C5\%84.pdf

Jupowicz-Ginalska, A., Kisilowska, Jasiewicz, J., M., Baran, T., and Wysocki, A. FOMO 2019. Polacy a lęk przed odłączeniem. Warszawa: WDIB UW. Available at: https://www.wdib.uw.edu.pl/attachments/article/2535/FOMO\%202019_Raport.pdf

Przybylski, A.K., Murayama, K., DeHaan, C.R., and Gladwell, V. (2013). Motivational, emotional, and behavioral correlates of fear of missing out. Computers in Human Behavior, 29(4), 1841-1848.

Reinecke, L., Aufenanger, S., Beutel, M.E., Dreier, M., Quiring, O., Stark, B., ... and Müller, K.W. (2017). Digital stress over the life span: The effects of communication load and internet multitasking on perceived stress and psychological health impairments in a German probability sample. Media Psychology, 20(1), 90-115.

Salim, F., Rahardjo, W., Tanaya, T., and Qurani, R. (2017). Is self-presentation of Instagram users influenced by friendship-contingent self-esteem and fear of missing out. Makara Hubs-Asia, 21(2), 70-82.

Scalzo, A.C. and Martinez, J.A. (2017). Not all anxiety is the same: How different "Types" of anxiety uniquely associate with college students' drinking intentions. Journal of College Student Development, 58(6), 943-947.

Xie, X., Wang, Y., Wang, P., Zhao, F., and Lei, L. (2018). Basic psychological needs satisfaction and fear of missing out: Friend support moderated the mediating effect of individual relative deprivation. Psychiatry Research, 26, 223-228.

Yin, F.S., Liu, M.L., and Lin, C.P. (2015). Forecasting the continuance intention of social networking sites: Assessing privacy risk and usefulness of technology. Technological Forecasting and Social Change, 99, 267-272. 